\title{
Germanica
}

\section{Abstrakte Oper Nr. 1 de Boris Blacher (1953), un Zeitoper expérimental?}

Eine experimentelle Zeitoper ? Boris Blachers Abstrakte Oper Nr. 1 (1953)

\section{Bernard Banoun}

\section{OpenEdition}

\section{Journals}

Édition électronique

URL : http://journals.openedition.org/germanica/475

DOI : $10.4000 /$ germanica.475

ISSN : 2107-0784

Éditeur

Université de Lille

Édition imprimée

Date de publication : 1 décembre 2007

Pagination : $91-112$

ISBN : 978-2-913857-20-9

ISSN : 0984-2632

Référence électronique

Bernard Banoun, «Abstrakte Oper Nr. 7 de Boris Blacher (1953), un Zeitoper expérimental ? ", Germanica [En ligne], 41 | 2007, mis en ligne le 01 décembre 2009, consulté le 06 octobre 2020. URL : http:// journals.openedition.org/germanica/475 ; DOI : https://doi.org/10.4000/germanica.475

Ce document a été généré automatiquement le 6 octobre 2020.

(c) Tous droits réservés 


\title{
Abstrakte Oper Nr. 1 de Boris Blacher (1953), un Zeitoper expérimental ?
}

Eine experimentelle Zeitoper? Boris Blachers Abstrakte Oper Nr. 1 (1953)

\author{
Bernard Banoun
}

1 Dans l'œuvre d'art plurimédiale qu'est l'opéra, le livret occupe une place singulière dans la chronologie d'élaboration de l'œuvre et ambivalente dans la hiérarchie des composantes : il est généralement le substrat sur lequel est composée la musique, l'élément fixe et stable sémantiquement, quand bien même la musique et la représentation brouillent la perception directe du sens qu'il véhicule. C'est également lui qui fournit le niveau le plus immédiatement saisissable de ce qu'on peut appeler « le dramatique dans l'opéra $»^{1}$, même si la dramaturgie musicale implique que la progression de l'intrigue soit retardée et interrompue par la présence d'éléments narratifs et introspectifs, bien plus souvent que ce n'est le cas au théâtre parlé. C'est là une constante depuis les commencements de l'opéra, une caractéristique due à la coprésence de plusieurs arts. Les choses se présentent différemment, surtout au $\mathrm{xx}^{\mathrm{e}}$ siècle, quand est pris pour modèle structurel un art qui n'est pas toujours directement constitutif de l'opéra : il en va ainsi du cinéma, dont les possibilités techniques influent sur la dramaturgie - Hin und zurück de Paul Hindemith, Intermezzo de Richard Strauss sont impensables sans le cinéma ${ }^{2}$. Quant aux rapports entre musique et arts plastiques, ils sont également anciens, avec notamment la musique à programme et les métaphores picturales utilisées par maints compositeurs pour décrire la musique ${ }^{3}$.

Cependant, une rupture novatrice est constituée par l'art abstrait dans ses rapports avec le drame lorsque Kandinsky et Schoenberg parviennent à produire une tension maximale entre le statique et le dramatique en construisant des actions scéniques dont le sens n'est pas porté par une intrigue mais avant tout expression d'une sensation ou d'une émotion, avec un recours minimal à la mimesis, comme dans Die glückliche Hand et Der gelbe Klang. On assiste là à l'irruption de l'abstraction dans le drame. L'opéra de Boris Blacher Abstrakte Oper Nr. 1 soulève, de ce point de vue, des questions particulièrement instructives justifiant une étude de détail. Après une présentation brève de ce compositeur injustement écarté de la mémoire des interprètes et du public 
et de certaines de ses œuvres, une description analytique d'Abstrakte Oper Nr. 1 cherchera à mettre en évidence les rapports entre action scénique et signification en soulignant la place paradoxale qu'y occupe le livret, ce qui permettra de s'interroger sur les rapports de cette œuvre avec le Zeitoper (opéra d'actualité).

\section{Situation historique de Blacher}

3 Alors que l'œuvre de Blacher dans son ensemble se caractérise par une grande flexibilité stylistique, le propos d'écrire cet "opéra abstrait» semble aller vers une réduction des moyens et du sens, une sorte de neutralité dans le style. Un aperçu sur le parcours de Blacher permet de mieux saisir les enjeux 4 . La flexibilité et l'apparent éclectisme de l'œuvre de Boris Blacher (1906-1975) étaient sans doute le fruit de ses expériences de jeunesse, puisqu'il avait reçu une éducation polyglotte et vécu dans divers pays d'Asie avant et après la Première Guerre mondiale; Blacher était accoutumé à une pratique musicale faite parfois d'expédients; ainsi avait-il été chargé par l'orchestre symphonique de Charbin en Chine, en l'absence de matériel d'orchestre, d'instrumenter des pièces à partir des seules réductions pour piano, par exemple la partition entière de Tosca5. Par la suite, ses études à Berlin, où il vécut de 1922 à sa mort en 1975, sont l'occasion de découvrir une production musicale abondante et, son père lui ayant coupé les vivres, c'est notamment un travail de transcripteur, d'arrangeur et de copiste qui fut son gagne-pain, dans une métropole où la mode du jazz et de la musique légère était soutenue par les nouveaux moyens de reproduction du son utilisés parfois par l'avant-garde 6 . Artiste refusant toute attitude et toute pose "romantiques ", Blacher ne cherche jamais la complexité pour elle-même, et si sa musique n'est ni de facture simple ni facile d'exécution, les moyens employés ne doivent pas entraver la compréhension du public. La brièveté de la forme et la vivacité du rythme aiguisent la perception de l'auditeur, qui n'est jamais entraîné par la seule mélodie ni baigné par des contours flous. On retrouve ainsi fréquemment à son propos des qualificatifs souvent employés au sujet de la Nouvelle Objectivité des années 1920: sachlich, mechanisch, rational, zweckmäßig7.

Dans les années d'entre-deux-guerres, l'esthétique de l'opéra s'était constituée par opposition à Wagner et aux compositeurs postromantiques. Après la Deuxième Guerre mondiale, l'esthétique musicale dominante sous le nazisme devient l'ennemi à combattre sous deux formes: d'une part un retour en force de la monumentalité, de la lourdeur et de l'emphase ; d'autre part la vogue d'une musique légère (d'opérette et de cinéma en particulier) sentimentale et redondante, signifiant avec insistance ce qu'elle veut exprimer. L'économie de moyens, dans la musique de Blacher plus encore que chez celle de la plupart de ses contemporains, répond au besoin de faire table rase de l'esthétique nazie 8 . Après 1945, Blacher reste à Berlin. Attentif et critique envers la situation politique et la politique culturelle berlinoise, il devient l'un des personnages les plus en vue de la vie culturelle à l'Ouest ${ }^{9}$; professeur de composition à la Hochschule für Musik (où l'on compte notamment parmi ses élèves Gottfried von Einem, George Crumb, Claude Ballif et Aribert Reimann), il succède en 1953 à Werner Egk à la tête de cette institution.

5 Il fréquente cependant les milieux artistiques de la zone soviétique, écrivant par exemple pour Ernst Busch et pour l'actrice et chanteuse Kate Kühl des chansons sur des textes de Brecht, Weisenborn, Piscator, Kästner, (r)établissant ainsi une continuité 
entre la République de Weimar et l'après-guerre. Correspondant à l'Ouest de l'Akademie der Künste de Berlin-Est, il est également lié à Paul Dessau (qui dédia à sa mémoire son septième quatuor, au premier mouvement intitulé "Trauer um Blacher »). Un autre témoignage de ses contacts à l'Est est sa contribution à la Jüdische Chronik, dont il composa le prologue ${ }^{10}$. Blacher est donc porteur de deux situations historiques conflictuelles et entrecroisées de la vie politique et culturelle de l'après-guerre : le lien avec les années 1919-1933 d'une part ; la partition Est-Ouest à Berlin, en Allemagne et dans le monde d'autre part.

\section{Blacher et l'opéra avant Abstrakte Oper Nr. 1}

6 L'intrigue de Habemeajaja (1929), premier opéra de Blacher, découvert et représenté en 1987, opéra de chambre sur un texte de " Haggars », pseudonyme d'Albert von Haller ${ }^{11}$, peut être rapidement résumée : le représentant d'une nation occidentale rend visite à un prince esquimau, Habemeajaja ; il espère gagner sa confiance pour exploiter les ressources de son pays et lui présente pour le séduire une radio; le prince vient en visite officielle en Occident, la presse et la bourse réagissent en conséquence, mais il s'avère que le prince n'est venu que pour retrouver la cantatrice dont il a entendu la voix à la radio. Habemeajaja s'apparente ainsi à des ouvrages contemporains tels que Jonny spielt auf de Krenek, archétype de l'opéra d'actualité qui mêle aux conventions de l'opéra des emprunts à la revue et à la comédie musicale et utilise le jazz, une caractéristique qui se retrouve dans Abstrakte Oper Nr. 1. Le livret participe de l'entreprise de dépoétisation de la langue des textes mis en musique qui caractérise ces années-là̀ $^{12}$ : il y est question des médias, de la bourse, d'impôts, de crise gouvernementale, etc. Comme bien d'autres Zeitopern, celui-ci met en scène des personnages contemporains de l'époque où l'œuvre est composée, des situations quotidiennes et liées à cette époque, ce qui lui confère une teneur véridique loin des conventions de l'opéra traditionnel. Les sujets ne sont plus puisés dans la mythologie, ce ne sont pas non plus des adaptations d'œuvres littéraires; au contraire, le journalisme envahit la scène d'opéra. Le Zeitoper s'accompagne souvent d'une visée pédagogique car il traite de débats qui concernent le commun des mortels, dût-il aborder des sujets jugés indignes du genre lyrique ${ }^{13}$ : l'argent, les affaires, le divorce, sont des thèmes privilégiés de ces opéras créés sous la République de Weimar. Stylistiquement aussi, l'opéra d'actualité cherche à se rapprocher du public en renonçant au moins superficiellement au style noble et en proclamant ouvertement le refus de la transcendance et d'un art étranger au quotidien. Comme bien des Zeitopern, le premier ouvrage scénique de Blacher repose sur le paradoxe de la plupart des œuvres de ce type : il applaudit à la modernité et se nourrit d'elle, et dans le même temps, il opère une critique d'un monde asservi aux biens matériels et incapable de dépasser l'horizon du quotidien. Certaines caractéristiques de Habemeajaja se retrouvent dans Abstrakte Oper Nr. 1, qu'il conviendra d'évaluer en fonction de son rapport à l'« actualité ».

Dans les années 1943-55, pas moins de dix ouvrages de Blacher pour la scène (ballets et opéras) sont composés et créés. Comme avant-guerre, le rejet de l'illustration sonore, le refus de chercher à produire une ambiance caractérisent sa production. Blacher compose en 1943/44 l'opéra de chambre Romeo und Julia (1943), « librement adapté de Shakespeare " (frei nach Shakespeare); il choisit ainsi un sujet qu'on peut considérer 
comme un archétype du romantisme et qui a déjà inspiré, entre autres, Bellini et Berlioz; mais au lieu d'aller dans le sens d'un épanchement lyrique, il distille le lyrisme jusqu'à une réduction extrême et cherche à créer une distance entre la musique et l'action. Ce recours à un mythe littéraire est à rapprocher du travail de Hans Werner Henze sur l'histoire de Manon Lescaut dans Boulevard Solitude; les deux compositeurs ne reprennent que quelques moments clés du drame, dont ils éliminent les ingrédients romantiques et plusieurs personnages secondaires.

Die Flut (1946) est le premier ouvrage scénique composé par Blacher après la guerre. Face à une situation de pénurie, notamment au théâtre, il faut écrire des pièces ne requérant pas de moyens excessifs. Faisant de nécessité vertu, Blacher donne dans ces années des ouvrages qui, soit sont destinés à la radio, soit exigent peu de décors. D'entrée de jeu, de tels ouvrages fonctionnent contre l'illusion scénique, puisqu'on ne saurait songer à un grand luxe de décor. La distanciation par réduction des moyens scéniques est d'abord déterminée ici par la situation matérielle. Premier opéra représenté en Allemagne après la guerre, La Marée fut un grand succès. Sa conception dramatique annonce Abstrakte Oper Nr. 1, mais sans une réduction aussi radicale des moyens. Il s'agit d'une parabole exprimant le pessimisme d'une génération sacrifiée : l'action se déroule sur un banc de sable près de l'épave d'un voilier échoué, " aujourd'hui ou hier " (heute oder gestern). Quatre personnages sont en scène : conduits par un pêcheur, un jeune homme, un banquier et sa jeune maîtresse se rendent sur la plage près d'une épave. La marée les surprend et les quatre personnages sont en danger; le banquier, convaincu du pouvoir illimité de l'argent, offre de l'argent au jeune homme pour qu'il aille à terre chercher un bateau; sa maîtresse, quant à elle, s'éprend du pêcheur, être simple, non corrompu par l'argent et la civilisation. Mais la marée redescend, les personnages sont sauvés, le jeune homme tue le banquier et prend son argent; c'est lui que suit la jeune fille, abandonnant le pêcheur. Le sujet possède une dimension symbolique et atemporelle, le caractère quasi existentialiste de l'intrigue éloignant l'ouvrage des Zeitopern des années $1920^{14}$. L'ouvrage révèle une tendance caractéristique de l'opéra d'après-guerre dans la mesure où il est redevable à la technique de l'opéra épique des années 1920 : la volonté d'objectivité, de neutralité y est fondée sur la réduction et la sobriété de la formation instrumentale (flûte, clarinette, basson, trompette, tuba, cordes), accompagnant l'action mais ne se permettant aucune incursion dans l'intériorité des personnages ; on hésite à vrai dire à parler de personnages, car il s'agit de types représentant, comme dans un oratorio, des êtres dans une situation précise. Dramaturgiquement également, cet ouvrage conçu pour la radio s'apparente à l'opéra épique, dans la mesure où le chœur qui donne les indications scéniques et commente l'action joue un rôle narratif comparable à l'usage des panneaux chez Brecht et Weill.

9 L'opéra suivant, Preussisches Märchen (1952), se distingue fortement des deux ouvrages qui l'encadrent chronologiquement et ne sera que brièvement évoqué ici. Inspiré du Capitaine de Köpenick de Carl Zuckmayer, il en reprend l'intrigue, une satire de la petitebourgeoisie et de la fascination pour l'uniforme des fonctionnaires et militaires dans l'Allemagne wilhelmienne. Blacher voulait écrire un opéra capable d'entrer de manière durable au répertoire ${ }^{15}$ et opta pour une œuvre de pur divertissement. S'il recourt à certains procédés de distanciation (par exemple en confiant le rôle du père Fadenkreutz à une femme, celui de sa femme à un homme, ou avec l'intrusion de la voix parlée dans des passages mis en musique), ces procédés renvoient à des conventions du comique à l'opéra. En outre, la couleur locale historique (le tournant du siècle), évoquée 
par les musiques de danse et les costumes, rapproche l'ouvrage de l'opérette à grand spectacle, hors de toute dialectique entre présent et passé telle que Brecht l'avait tentée dans Die Dreigroschenoper. Certes, ces personnages crédules, pantins malléables, pouvaient trouver une sinistre résonance dans l'Allemagne de l'immédiate aprèsguerre, mais le charme facile de la musique la cantonne dans un espace de représentation où se mêlent surtout la nostalgie du Berlin de 1900 et l'amusement.

\section{Abstrakte Oper Nr. 1} rendre compte sur une scène d'opéra de la situation de l'homme moderne en rompant avec l'opéra traditionnel. Durant l'hiver 1952-1953, le compositeur Werner Egk, avec lequel Blacher était lié depuis l'avant-guerre, voulut tenter de trouver un équivalent scénique et musical à la peinture abstraite et proposa à Blacher un livret ${ }^{16}$. Commande de la radio hessoise, l'ouvrage fut créé sous forme concertante le 28 juin 1953 et scénique, à Mannheim, le 17 octobre de la même année. La création de cet opéra d'une demi-heure environ fut l'un des plus violents scandales du théâtre allemand d'aprèsguerre $^{17}$.

En peinture, les objets, les personnages et le « réel » ont disparu au sens où ils ne sont plus représentés ou figurés sur un principe de mimesis. Egk constate qu'après plusieurs décennies d'abstraction en peinture, l'opéra est en retard. Même les expérimentations et ouvrages d'avant-garde des années 1920 conservent le principe d'une action scénique animée par de vrais personnages de théâtre s'exprimant dans une langue traditionnelle ${ }^{18}$. Il serait pourtant envisageable selon Egk d'écrire un opéra sans « sujet » fourni par le texte, c'est-à-dire que l'élément le plus immédiatement signifiant à l'opéra, le verbe, serait conservé formellement mais privé de sens, ce qui transpose dans le domaine du livret d'opéra certaines des expérimentations verbales et poétiques des expressionnistes et de l'entre-deux-guerres.

Le titre du livret conçu pour Blacher est significatif. « Opéra » renvoie ici uniquement à un mode d'expression artistique. " $N^{\circ} 1$ » est d'une part une allusion, sérieuse ou ironique, aux titres souvent donnés à des tableaux d'art abstrait: titres généraux évitant toute référence à un contenu, une représentation ou un message, tels que « composition », «figure », "sans titre », etc., suivis d'un numéro à l'intérieur d'une série. D'autre part, le numéro fait penser à un premier essai destiné à être poursuivi - si rien n'indique que Blacher et Egk aient jamais envisagé un Opéra abstrait $n^{\circ} 2$, le numéro souligne l'aspect expérimental du projet. L'abstraction du texte ne signifie pourtant pas que l'ouvrage soit atemporel et dépourvu de signification historique. Au contraire, Abstrakte Oper Nr. 1 exprime à sa manière la situation de l'homme dans l'après-guerre, le sentiment d'une aliénation totale et d'une communication mensongère ou absente, une angoisse devant un danger diffus planant sur l'humanité livrée à une histoire comme catastrophe permanente. Comme le formulait Adorno à l'époque à propos de la peinture abstraite, l'absolue pureté de l'œuvre abstraite fait sa différence absolue avec le réel, mais sans le nier; elle apparaît comme la seule possibilité d'expression dans l'aveu de son imperfection, c'est-à-dire son incapacité à rendre compte du réel de manière plus directe et adéquate ${ }^{19}$.

Dans l'avant-propos à l'édition de 1973 de la partition, le «librettiste » Werner Egk spécifie ceci : «Dem Werk liegt die Idee zugrunde, eine Reihe von für unsere Zeit und 
unser Erleben typischen Grundsituationen einzufangen und in einer abstrakten Form, d.h. ohne zusammenhängende Handlung und mit Hilfe eines abstrakten Textes zu gestalten. Die komponierten Worte sind musikalisch-phonetisch erfunden und richten sich an das automatische Assoziationsvermögen des Zuhörers. ${ }^{20} "$ Comme le rapporte Egk dans ses mémoires, le metteur en scène de la première exécution scénique, Hans Schüler, appliqua aux décors un principe analogue: "Die Vorgänge laufen wie im Traum mit der für ihn typischen Lebendigkeit, jedoch im Vergleich zur Kausalitätskette einer wirklichen Handlung in veränderter und verschwimmender Form ideenfluchtartig ab. ${ }^{21}$ »

Le «livret» d'Abstrakte Oper Nr. 1 se présente comme une suite de sept scènes. À l'exception de la scène 4 , il ne comporte ni action ni texte au sens conventionnel des termes. Nul dialogue logique et articulé, mais des phonèmes sans signification, d'une ou plusieurs syllabes. Le texte peut certes être qualifié de livret et Egk de librettiste de Blacher dans la mesure où ce dernier a mis son texte en musique; cependant, le spectateur n'a nul besoin d'emporter dans sa poche au théatre le libretto pour suivre l'action, puisque l'action n'est pas portée par les mots comme unités minimales du sens dans une phrase, des dialogues et des scènes qui les articulent selon la logique d'une langue et d'une fable.

Si la dimension sémantique n'est pas totalement absente, comme on le verra, il ne s'agit pas de signifiants connus. En effet, les mots sont, comme l'écrit Egk dans la citation donnée précédemment, "des inventions musico-phonétiques [qui] s'adressent au pouvoir d'associations spontanées de l'auditeur ». Il s'agit, à l'exception de la scène 4, de mots inventés, telles les glossolalies d'Antonin Artaud, les inventions d'Henri Michaux ou Kurt Schwitters, mais non d'un substrat textuel définitif. Les mots sont ici à géométrie variable, ils apparaissent comme réservoir de sonorités livrées au compositeur qui peut y puiser à sa guise, les inverser et les combiner ${ }^{22}$. L'unique contrainte provenant du texte est le regroupement par scènes : pour chacune des sept scènes, le réservoir de sonorités est différent, avec prédominance de telles ou telles voyelles et consonnes $(a-u ; u-u ̈$, etc. dans la scène 1$)$, de telle ou telle assonance destinée à provoquer une association chez l'auditeur, ce principe apparaissant le plus clairement dans la scène 5 .

Le texte fourni par Egk ne comporte pas de liste de personnages, ni d'indications sur le lieu et l'époque de l'«action»; il n'y a également aucune didascalie (sauf dans l'introduction au livret); Egk se contente d'indiquer l'ordre des sept scènes qui composent l'opéra: scène 1: Angst; scène 2 : Liebe ; scène 3 : Schmerz; scène 4 : Verhandlung; scène 5 : Panik; scène $6:$ Liebe II ; scène $7:$ Angst. Il n'y a pas d'intrigue au sens conventionnel, mais chaque scène est donc tout de même rattachée à une situation ou à un état psychologique; l'absence de contenu est en outre compensée par la construction de l'ensemble, qui est signifiante. Les sept scènes sont disposées en miroir. L'unique scène dont le texte soit à première vue porteur de sens est la scène 4, Négociation; autour d'elle gravitent des scènes symétriques, Panique étant symétrique de Douleur et, on le verra, évoquant une atmosphère analogue mais plus générale et plus terrible. 


\section{La musique}

Il existe deux versions de l'ouvrage, l'une de 1953, l'autre de 1957, qui se différencient par le nombre d'instruments par pupitre (réduit généralement de moitié dans la seconde version). L'instrumentation est dérivée d'un big-band de jazz et comprend clarinettes, clarinette basse, saxophone ténor, trompettes, tubas, piano, contrebasse, timbales et percussions. Elle est donc réduite par rapport à celle de l'orchestre symphonique encore requis par Blacher dans Conte prussien; elle ne comprend pas de cordes (à l'exception de la contrebasse, instrument soliste en jazz).

Selon un paradoxe fructueux depuis Pierrot lunaire, cette formation instrumentale déjà très réduite n'est pas nécessairement utilisée dans son ensemble à chaque scène. $\mathrm{Au}$ contraire, les instruments sont dissociés d'une scène à l'autre et leur timbre sert à en exprimer l'ambiance; il est associé à telle ou telle situation et se voit donc pourvu d'une fonction sémantique. Les connotations associées à tel ou tel instrument ou groupe d'instrument livrent à elles seules le décor et le contenu de la scène : c'est le cas dans la majorité des opéras, avant même que le texte ne commence, et $a$ fortiori dans cet opéra en particulier, puisque le texte n'apporte pas d'information rationnelle. Quant aux chanteurs, Blacher a écrit l'opéra pour une configuration très simple (soprano, ténor et baryton - ce qui n'est pas sans évoquer ironiquement les configurations vocales chez Donizetti et le premier Verdi) à laquelle s'ajoute un petit chœur mixte.

\section{Description et analyse des scènes}

\section{Scène 1}

Le matériau verbal de la scène 1 consiste en des voyelles $(a-u, a-u ̈, a-o-u)$ ainsi que, pour le chœur, les syllabes « agattagatta ».

L'opéra ne comporte pas d'ouverture séparée mais commence par vingt-huit mesures instrumentales, véritable décor sonore. L'angoisse qui donne son titre à la scène est associée immédiatement à la guerre, avec des roulements de tambours (tambourin, grosse caisse et timbale) d'abord pianissimo entrecoupés d'accents forte. Il n'y a donc aucun instrument mélodique, et les sonorités militaires dominent.

Quant au chant, il est associé d'emblée au cri, avec un mi initial de la soprano sur la voyelle $a$; le chœur, par la suite, livre un arrière-plan sonore haletant. Des descentes chromatiques et l'alternance entre les intervalles de secondes majeures et mineures sur les sons a-au expriment la plainte. C'est presque ici une expression codée. La descente chromatique exprime souvent, dans la musique occidentale tout au moins, la déploration et la plainte : ainsi dans le passage instrumental qui précède la mort de Didon dans Dido and Aeneas de Purcell, dans l'annonce de la mort d'Oreste dans Elektra de Strauss ou encore dans le Kyrie du Requiem de Krysztof Penderecki. Outre ces intervalles de demi-tons, on trouve plusieurs fois leur extension sous forme d'octaves descendantes : jamais d'octaves justes, mais des octaves diminuées ou augmentées, chez les solistes (exemple: mesures 224-22623). On peut donc parler ici d'un aspect sémantique de la musique comme langage expressif, fût-il fondé sur des conventions et $\operatorname{codes}^{24}$. 
scène assez brève ne peint qu'une seule atmosphère, il n'y a pas de crescendo continu ni de climax ; amputée de mesures conclusives, elle s'interrompt brutalement.

\section{Scène 2}

Si la scène 1 en elle-même n'a pas de "contenu » et recourt uniquement à certaines associations avec des sonorités instrumentales ou des usages de la voix liés à la peur, la scène 2, par contraste, vient pour ainsi dire préciser rétrospectivement le sens de la première scène. Exemple de l'utilisation des pupitres dissociés, elle s'ouvre sur un bref motif confié à la clarinette et à la clarinette basse, relayées par le saxophone.

Blacher utilise en particulier la mélodie et la rondeur des timbres pour traduire le titre donné à la scène, Amour I. Lui et son librettiste recourent à certaines conventions de l'opéra, le duo d'amour, qu'ils exploitent et parodient tout à la fois. En effet, le duo d'amour traditionnel présente d'intéressantes analogies avec le rapport entre le texte et la musique tel que les auteurs l'ont ici imaginé. Le duo d'amour romantique, par exemple dans Tristan und Isolde ou dans l'opéra italien, est par excellence le lieu où le sens des mots peut être relayé par la musique; le texte en est souvent bref, les répétitions nombreuses; il sert l'épanchement lyrique, auquel suffisent des syllabes qui pourraient n'avoir aucun sens. Ainsi, le « doux petit mot Du» (dies süße Wörtlein Du) répété tant de fois par Tristan, ou les ma et ta de « m'ami » et «t'amo » dans un duo tel que celui d'Un Ballo in maschera de Verdi à l'acte II sont uniquement les point de départ d'une expansion du chant qui se suffit bientôt à lui-même; les syllabes sont les supports sonores de l'ineffable. Il n'en va guère autrement dans cette scène II. Egk écrivit un texte abondant en consonnes $b$ et $l$ (La ga Ba ba La ga Ba bu, etc.) chanté par le ténor et le soprano, babil auquel répond un roucoulement au sens propre du terme : "O Curru Curru Curru », chante le ténor souvent sur une même note, comme un chant d'oiseau transcrit par le compositeur.

Après ces éléments quasi parodiques servant notamment à fixer le sens par les associations qu'ils suscitent, Blacher écrit une mélodie légèrement ornementée, le premier passage réellement cantando de cet opéra, auquel la soprano répond par un motif chromatique sur les syllabes " $O$ gaga o gaga ». C'est là comme la réduction minimale d'un dialogue d'amour à l'opéra, dont Egk et Blacher donnent ici les signes conventionnels, ponctué d'unissons (des mi fortissimo sur la voyelle $a$ aux mesures 53 et suivantes) ${ }^{25}$.

On note enfin dans cette scène la fonction impartie au jazz: Blacher n'écrit pas littéralement du jazz - rien n'est laissé à l'improvisation -, mais en reprend certains éléments isolés à des fins de sémantisation ${ }^{26}$. Il ne s'agit pas comme chez Debussy, Stravinsky ou Weill d'insuffler une nouvelle vie à la musique par un exotisme des rythmes et des harmonies. Chez Blacher, les emprunts au jazz (saxophone, rythmes, etc.) sont déjà au second degré; déjà connoté, le jazz renvoie à une entité géographique, historique et symbolique à la fois, les Etats-Unis.

De ce point de vue, cette scène est instructive quant à l'abstraction d'Abstrakte Oper Nr. 1 ; l'amour évoqué par le jazz n'est pas un sentiment hors du temps ; si impersonnels que semblent être les rapports entre le soprano et le ténor, Blacher évoque ici moins l'amour en général, s'il existe, que des « lieux d'amour » dans la société allemande déjà très américanisée dans ces années de plan Marshall. L'image de l'Amérique est alors 
double : le jazz évoque la vitalité et la liberté de la grande ville, mais aussi l'anonymat et la grisaille du quotidien ${ }^{27}$.

\section{Scène 3} aussi exception d'une autre manière: premièrement, son titre fait référence à une situation ou un processus et non à un sentiment ou une atmosphère; deuxièmement, son substrat textuel est formé de mots du dictionnaire. Cependant, leur contenu est si peu fixé que le compositeur peut leur donner les inflexions de son choix. De plus, cette apparition subite de mots oblige à relativiser, on le verra, les rapports entre, d'une part, des syllabes dénuées de sens et la musique, d'autre part des répliques écrites en langue naturelle. Cette scène provoque un jeu très subtil sur les éléments porteurs de sens, sur les possibilités sémantiques des diverses composantes du théâtre musical. Elle est confiée aux deux voix d'homme. Le ténor chante en anglais, le baryton en russe, les textes suivants. La transcription de l'alphabet cyrillique ci-dessous est celle qui figure dans la partition; il en va de même des erreurs, peut-être volontaires, en anglais (perminent à la place de permanent ; wether à la place de weather) et de la ponctuation, qui semble tout à fait fortuite. En vérité, il serait plus juste d'écrire entre guillemets que le texte est en «russe » et en "anglais » ou "américain», ce qui soulignerait le côté fabriqué, l'indigence extrême de ces lignes qui renvoient à l'Union soviétique et aux États-Unis avec des traits volontairement grossiers ${ }^{28}$.

\section{Texte en russe :}

Patschemu otwetschai

Patschemu

Patschemu

Da, otwetschai $[. .$.

Sluschaite otwetschai, otwetschai. Otwet patschemu. ach Da. Patschemu otwetschaite. Pagoda, charoscha. Ach da, sluschaite, etc.

Ce texte pourrait être, en substance, rendu ainsi :

Pourquoi, réponds, oui, réponds, écoute, toi, réponds ${ }^{29}$. Une réponse. Pourquoi, ah oui, pourquoi. Il fait beau. Ah oui, écoute. 

L'Américain répète un « O.K., O.K. » stéréotypé. Le seul lien explicite avec le titre de la scène est "I hope you have met him ", qui signale des tentatives de dialogue entre les secteurs soviétique et américain. Mais l'échange ne va guère plus loin, puisque l'Américain dit tantôt une chose, tantôt son contraire ("Statements were made, statements were not made »), pour se replier bientôt sur des banalités ("weather is fine »). Les deux hommes « dialoguent » donc sans interprète, mais la musique ne parvient pas à établir une autre communication.

\section{Scène 5}

La scène 5, Panique, est la plus longue de l'opéra (278 mesures), c'est aussi la plus complexe sur le plan de l'écriture vocale et chorale. Elle apparaît ainsi, plus que la scène 4 , comme le centre de gravité de l'œuvre. En outre, le chœur occupe ici la plus 
grande place, il ouvre la scène et intervient sans cesse, comme si les pourparlers laissés en suspens à la scène précédente ne faisaient que renforcer l'angoisse des populations.

Le texte de cette scène est à mi-chemin entre les syllabes dépourvues de sens et les mots du dictionnaire. Ici, Egk a voulu trouver ce qu'il appelle dans sa note introductive des «mots de panique" évoquant délibérément l'état d'esprit qui règne après Hiroshima, grâce à l'abondance de sonorités telles que $z$ et $d z$, et surtout de syllabes empruntées évoquant des noms d'explosifs: «azidazant, apodalip, adynazit, anitronit ${ }^{31}$ ». Un jeu sur le mot inventé mais dont les connotations n'en sont pas moins perceptibles se retrouve aussi dans " akaplozit », qui pourrait être un anagramme d'" apocalypse » ou un mélange des mots « apocalypse » et " explosion ». Le texte recourt donc à la fonction poétique du langage, d'une manière quasi dadaïste, mais il ne s'agit pas seulement d'un usage ludique : la perte du sens s'accompagne ici d'une déshumanisation, le langage étant uniquement celui de la technique et de la chimie.

Cette scène est aussi musicalement la plus complexe de l'opéra. Cependant, elle n'en possède pas moins une structure clairement perceptible. Elle commence par un crescendo atteignant un climax fortissimo, où le chœur vocalise en un fugato assez complexe relayé par les clarinettes (mesures 150 à 173), avant une reprise pianissimo. À ce développement linéaire se superpose une structure ternaire, car chaque séquence est elle-même un crescendo, et le fortissimo atteint conduit à l'intervention d'un soliste a cappella, chromatique et plaintive : le soprano (mesures 80 à 95), le baryton (mesures 174 à 188), puis le ténor (mesures 251 à 274), dont les derniers mots, accompagnés puis prolongés par des octaves au piano et des coups de timbales pianissimo ont quelque chose d'une lugubre défaite sinon d'un total anéantissement. Le sens de ce passage est également donné par l'écriture musicale : le fugato déjà mentionné, avec des vocalises évoquant par exemple une cantate de Bach, semble vouloir dire que la technique meurtrière règne désormais; les mots, intelligibles au début, ne le sont plus, mais ce chœur traduit à la fois une exaltation et un effroi collectifs ${ }^{32}$.

\section{Scène 6}

40 Le passage de Panique à Amour II se fait sur un mode analogue à celui d'Angoisse à Amour I. La couleur sonore change, l'écriture est plus dépouillée et presque transparente, le chœur est moins fourni : le seul soliste est le ténor, accompagné par un chœur de femmes (alti et soprani). Le schéma de base à trois temps évoque une valse d'opérette et de comédie musicale : la musique est ici délibérément « culinaire » (au sens que Brecht donnait à ce terme), le plaisir du chant est intact, d'autant qu'il est absolument inutile de comprendre le texte de la mélodie fredonnée dans l'insouciance par le ténor, un enchaînement de syllabes comptant très peu de consonnes dures et utilisant tour à tour et mécaniquement les cinq voyelles, telles que « la la na na lu lu nu nu - za za da da gaga, warawawawa - zezedederugegewewe, etc.» (Exemple: mesures 47 à 63). La scène est construite sur la forme lied ( $\mathrm{ABA})$, mais la répétition de la première partie (à partir de la mesure 100) n'est pas intégrale; la mélodie est reprise sur des valeurs différentes (noires et blanches) et devient plus calme.

41 Le contraste avec l'atmosphère de la scène précédente est renforcé par la pulsation rythmique qui évoque le jazz. Blacher utilise ici les mètres variables: partant de mesures $3 / 4$ puis $3 / 8$, il y revient sans cesse et la prédominance des mesures impaires donne au passage une irrégularité et une liberté qui tranchent avec le caractère 
oppressant de ce qui précédait (mesures 25 à 29, etc.) ${ }^{33}$. L'évocation du jazz provient aussi du rapport entre texte et musique : certaines phrases (mesures 130 et suivantes par exemple) ressemblent à du scat qui aurait été noté.

Le passage se clôt sur un sol aigu plein de liberté, mais les quatre accords ascendants du chœur, les pizzicati de contrebasse ainsi que les coups de timbales qui forment le soubassement de cet aigu final tempèrent la sérénité que dégage le jeune premier. La jovialité de l'atmosphère de la comédie musicale américaine est contrebalancée et mise en doute par des moyens musicaux: leur parcimonie est aux antipodes des cordes lyriques, sinon sirupeuses, qui pourraient soutenir un tel chant du ténor dans le genre d'ouvrages auxquels renvoie cette scène ; par là, Blacher utilise le principe du contraste entre une référence musicale clairement identifiable, codifiée, et une situation. Pris entre deux scènes dont les atmosphères sont très différentes, entre Panique et Angoisse, cet air du ténor qui demeure d'ailleurs solitaire traduit ainsi un bonheur d'autant plus précaire.

\section{Scène 7}

Intitulée Angoisse, la dernière scène n'est pas uniquement par son titre le symétrique de la scène 1 . Rendant très perceptible la construction de ce bref opéra, elle en reprend les éléments principaux, mais sans les interventions du chœur et avec une formation instrumentale plus réduite : piano, percussions et cuivres. Elle produit ainsi un effet d'écho et, dans la raréfaction du son, reconduit l'auditeur vers les roulements de tambour du début après quelques mesures où les trois solistes chantent ensemble, mais sans accompagnement, comme coupés du reste du monde et eux-mêmes étrangers l'un à l'autre.

\section{Musique, théâtre et signification dans Abstrakte Oper Nr. 1}

Abstrakte Oper Nr. 1, par son caractère expérimental, permet de préciser les rapports entre les différentes composantes du théâtre musical et la question de la signification de la musique dramatique. En effet, l'intrigue au sens courant du terme est laissée de côté et l'on pourrait croire que seule la musique et la scène sont en jeu, chaque tableau devant exprimer ou évoquer un état d'esprit ou une situation. Le choix des moyens offre des perspectives instructives sur les possibilités de l'opéra si on les considère, pour parler avec Brecht, en fonction du principe de la séparation des éléments. En effet, Egk et Blacher laissent de côté au moins deux éléments de l'opéra comme forme artistique regroupant plusieurs arts. Le verbe, ou plutôt la langue comme système de signifiants, n'a pas ici la place qu'elle occupe dans le théâtre musical traditionnel : cet opéra déclare d'avance forfait dans la « lutte pour la primauté » (der große Primatkampf), selon l'expression de Brecht caractérisant les rapports entre texte et musique ${ }^{34}$, puisqu'on n'a pas à comprendre le texte. Mais on a vu plus haut que ce refus du signifiant n'est pas respecté de bout en bout ; il demeure ambigu, puisque les scènes 4 et 5 , déformant des mots, exigent l'intelligibilité, car la distance avec la langue courante (que cette distance soit jugée parodique ou sérieuse) est l'un des éléments sur lesquels elles reposent. Mis à part dans ces deux scènes, on ne peut dire que le librettiste ait songé à inventer une nouvelle langue comme le faisait par exemple Kurt 
Schwitters dans un texte de 1925, Sprache, où il établissait une liste de monosyllabes destinés à être substitués à un ensemble de phrases essentielles : extérieurement, les syllabes de Schwitters sont les mêmes que chez Egk ( $b a=$ je suis ou j'ai ; $b o=$ je veux, etc. $)^{35}$, mais Egk exploite la musicalité de la langue sans se soucier du sens et laisse à Blacher le soin de sémantiser la musique. Les compétences sont donc interverties.

Semble en revanche nettement remise en question la fonction du livret comme texte théâtral sur lequel une musique vient s'articuler. Le « livret » de Werner Egk n'est pas un texte fixé obéissant à la logique du discours permettant la conduite d'une intrigue ayant une exposition et un dénouement. À l'intérieur de chaque scène, le texte est malléable et ouvert. Les deux auteurs laissent ainsi de côté un élément qui "pèse " toujours sur les rapports entre texte et musique à l'opéra, qui n'est pas seulement l'intelligibilité du texte (problème qui se pose aussi dans le lied, l'oratorio, etc.), mais la structuration de dialogues en vue d'une mise en musique n'entravant pas totalement la compréhension de l'intrigue. La suppression de cette contrainte fait que le sens est moins fixé et que l'ouvrage lui-même devient plus malléable ${ }^{36}$.

Le texte chanté et un sens qui doit être représenté sont dissociés, déconnectés. Cela ne ruine pas le précepte brechtien - incontestable en théorie mais dont les possibilités de réalisation pratique sont tout de même assez réduites et ne peuvent être employées systématiquement -, précepte selon lequel les éléments doivent se contredire.

$\mathrm{Au}$ contraire, le principe sur lequel est conçu Abstrakte Oper Nr. 1 permet à la représentation de varier fortement, il laisse une très grande liberté à celui qui est chargé de réaliser l'ouvrage scéniquement. Ainsi, lors de la création concertante à Francfort, des clowns venaient entre deux scènes et jouaient de petits intermèdes comiques $^{37}$ : un éclairage ironique était jeté sur les personnages, ce qu'on ne soupçonnerait pas à la lecture de la partition ou à la seule audition - en effet, les onomatopées, qui peuvent paraître dérisoires au début, ne le sont pas nécessairement lorsqu'elles sont chantées. L'opéra fonctionnait donc sur le principe de l'action sérieuse avec intermezzi comiques, chose fort courante dans l'histoire de l'opéra, surtout dans l'opéra italien des $\mathrm{XVII}^{\mathrm{e}}$ et $\mathrm{XVIII}$ e siècles. Seule une action juxtaposant des scènes assez brèves, et une mise en musique discontinue permet cette solution. Le théâtre vient donc enrichir le discours musical, par l'interruption et le contraste.

De même, Egk fournit dans sa brève introduction quelques indications scéniques : "In Liebe $^{38}$ bringt der Tenor eine Schneiderpuppe auf die Bühne und schmückt sie. Der Sopran liebt den Tenor und wird von ihm zurückgewiesen. Zum Schluß der Szene erschießt der Sopran die Schneiderpuppe. » Egk donne donc des éléments d'intrigue, mais ils ne sont liés de manière nécessaire et contraignante ni au livret ni à la musique ; la scène, troisième composante essentielle de l'opéra, peut donc apporter éventuellement un poids concret et « réaliste » au propos volontairement abstrait de la musique et du texte. L'abstraction a donc été transposée du domaine pictural ou visuel vers l'élément dramatique qui ne conserve que des bribes incertaines de sens.

\section{Désémantisation du livret et sémantisation de la musique}

Dans ses mémoires, Werner Egk, rappelant l'idée initiale d'Abstrakte Oper Nr. 1, décrit le texte ainsi : "Chiffrierte Handlungsrudimente, Lautfetzen, abstrakte Wortbildungen, 
ein formelhafter Dialog, dessen Bedeutung in seiner Formelhaftigkeit liegt, nicht in seinem Inhalt ${ }^{39}$. " Les premiers éléments de ce commentaire de l'auteur lui-même ont déjà été relevés plus haut: ils s'appliquent effectivement au texte et sont conservés dans sa mise en musique. Les derniers mots de cette description, en revanche, sont plus surprenants. En qualifiant le dialogue de "formel » et en en situant la signification dans ce caractère formel, Egk établit un parallèle entre texte et musique et inverse leur rôle. La langue comme système de signifiants passe au second plan. En revanche, chaque scène doit exprimer une ambiance ou produire une impression.

Dibelius rapproche rapidement les sept scènes de l'opéra de Blacher des trois mouvements de la Musique d'accompagnement pour une scène de film de Schoenberg (Begleitmusik zu einer Lichtspielszene, opus 34, 1930). Les trois pièces sont en effet intitulées Danger menaçant, Angoisse et Catastrophe. Schoenberg n'écrivit pas cette pièce pour un film réel, mais à la suite de la suggestion de l'éditeur Heinrichshofen qui avait demandé à plusieurs compositeurs des œuvres de ce genre sans qu'elles fussent liées à un film particulier. Ne serait-ce que par leurs titres, les pièces de Schoenberg font en effet songer à Blacher. Il s'agit de trois morceaux de musique dérivée de la période expressionniste du compositeur, d'une musique à programme, capable d'« exprimer ", de peindre des sentiments exacerbés et des situations extrêmes, comme dans Erwartung ou dans un bref poème symphonique. Le principe de base est la montée puis la baisse de la tension. On imagine les films que cette musique aurait pu accompagner : le cinéma allemand des années 1920, de Lang ou Murnau, en a produit un grand nombre. Cette œuvre de Schoenberg est d'autant plus intéressante si on la situe par rapport aux écrits de Brecht sur la musique de cinéma. C'est en effet, on l'a vu, dans sa réflexion sur la musique de cinéma que Brecht pose le plus clairement le refus de la redondance entre image, situation et musique et prône un contraste. Mais pour qu'un tel contraste soit perceptible, cela suppose que telle musique évoque telle situation ou tel sentiment. Cette œuvre de Schoenberg est une étude sur les possibilités expressives et dramatiques intrinsèques de la musique.

51 Schoenberg et Blacher explorent tous deux les rapports entre musique et signification. Chez Schoenberg, la musique est déconnectée de l'image à laquelle elle pourrait être associée tandis que chez Blacher, l'élément manquant est l'intrigue dramatique portée par un dialogue. Chez Schoenberg, l'alternance entre tension et détente est le principal élément expressif et constitue, hors des considérations sur l'écriture musicale et la forme de ces pièces, l'un des universaux de la musique; chez Blacher, les références sont plus ponctuelles, plus parcellaires, ou plus circonscrites historiquement. La réduction des situations à des données concrètes minimales ne contribue donc pas nécessairement à souligner un aspect a-historique de cet ouvrage qui s'apparente au Zeitoper.

\section{Abstrakte Oper Nr. 1 et le Zeitoper ${ }^{40}$}

«Le matin elle sautait de son lit très tôt, courait dans l'appartement, âcre, serrée, toute chargée de cris, de gestes, de halètements de colère, de 'scènes' » : ainsi commence un chapitre, le sixième, de Tropismes de Nathalie Sarraute, paru en 1939 et vraiment découvert dans les années $1950^{41}$. Dans cette œuvre jalon, dont la réception est quasi contemporaine de l'opéra de Boris Blacher, Nathalie Sarraute met en pratique sa conviction selon laquelle il n'est plus possible de produire des personnages au sens 
traditionnel du terme, des êtres dont on puisse raconter l'histoire dans son déroulement linéaire, en décrivant leur passé et leur traits comme ceux d'individus uniques; seuls des «il », «ils", « elle», « elles", peuplent ces pages, on devine qu'à chaque chapitre il peut s'agir d'un autre ou d'une autre, mais aucun nom propre ne vient fixer cette identité. Pourtant, on ne saurait dire que ce livre soit atemporel. Bien au contraire, plus d'un élément indique que ces personnages si «abstraits" appartiennent à une classe sociale donnée et ils sont saisis dans des instants précis de leur existence, instants identifiables. Avec le recul des années, Tropismes paraît singulièrement « réaliste » et même figuratif, quand bien même on ne voit nul visage ; c'est une expression concentrée des faits et gestes des Français de la classe moyenne dans les années 1950. Des situations types du quotidien dévoilent à la fois leur banalité et leur extrême violence. Les êtres ne se montrent plus avec l'habituelle épaisseur psychologique à laquelle nous a habitués le roman traditionnel, et malgré tout, la situation psychologique est très présente.

Ce rapprochement n'a ici de valeur qu'en ce qu'il éclaire peut-être ce qui dans l'opéra de Blacher est rapport entre l'abstraction et le présent. L'ouvrage se focalise sur certaines situations précises, chacune exprimant un sentiment et un seul, mais ces sentiments ne sont pas aussi abstraits que le titre pourrait le laisser croire. Certes, chaque scène est placée sous un certain éclairage, mais l'angoisse, la panique ou l'amour de l'homme ne sont pas identiques à toutes les époques. Même les scènes autres que la quatrième, la plus fixée historiquement, prennent leur densité dans l'après-Hiroshima; les sentiments exprimés ici sont situés historiquement et socialement.

Comme Tauben im Gras de Wolfgang Koeppen ou les vers célèbres du poème Alle Tage d'Ingeborg Bachmann - «Der Krieg wird nicht mehr erklärt,/sondern fortgesetzt ${ }^{42}$ »ou encore Boulevard Solitude de Hans Werner Henze, l'opéra de Blacher ne peut être isolé du contexte historique : ces opéras sont nés dans l'Allemagne d'après-guerre, peu avant les débats sur le réarmement, en pleine guerre froide. Si leur validité n'est pas restreinte à cette époque, ils tiennent cependant du Zeitoper. Le caractère expérimental du livret peut être rapproché des travaux de la Wiener Gruppe en Autriche à partir de la fin des années 1950: la langue est refusée comme un fait allant de soi, son dévoiement historique provoque un repli vers la sonorité pure et la mise à distance des signifiants, de manière à mettre en doute leur caractère naturel. L'abstraction n'est pas déconnexion avec le réel, elle est un moyen pour sortir l'art de sa compromission avec la politique sous le régime national-socialiste. Dans la Wiener Gruppe comme dans Abstrakte Oper Nr. 1, un langage signifiant (la langue allemande dans un cas, l'opéra comme œuvre destinée à la représentation dans l'autre cas) est coupé d'un lien immédiat au signifié. L'absence d'une intrigue continue, le recours à une langue mutilée, ne confrontent pas le spectateur à une expérimentation inintelligible. Même les lambeaux de paroles d'Abstrakte Oper Nr. 1 et sa musique au second degré sont en quelque sorte hyperréalistes. 


\section{NOTES}

1. Cf. Jean-Charles Margotton : «Le dramatique dans l'opéra ", in : Cahiers d'Etudes Germaniques 20 (1991), p. 149-160. Sur le caractère plurimédial du genre, voir notamment Albert Gier: Das Libretto. Theorie und Geschichte einer musikoliterarischen Gattung, Darmstadt, Wissenschaftliche Buchgesellschaft, 1998, p. 5.

2. Lulu de Berg présente le cas d'un usage réel et non métaphorique du langage cinématographique. Il faudrait par ailleurs évoquer l'influence de la radio sur la dramaturgie de l'opéra, et cela pas exclusivement dans le domaine du Funkoper.

3. Ainsi des Images de Debussy, des Tableaux d'une exposition de Moussorgsky, mais aussi des rapports avec les couleurs et la composition picturale chez Olivier Messiaen ou Michèle Reverdy.

4. Sur cette flexibilité créatrice et stylistique, notamment dans ses rapports au "postmoderne ", voir le précieux ouvrage de Stephan Mösch: Der gebrauchte Text. Studien zu den Libretti Boris Blachers, Stuttgart, Metzler, 2002, p. 3-7. - Le présent article provient d'un travail d'habilitation à diriger les recherches présenté en novembre 2000 à la Sorbonne; il est complété et modifié en tenant compte de publications récentes. Les principales sources, en plus des ouvrages cités, sont le fonds Blacher à l'Akademie der Künste (Berlin) et les archives des éditions Bote \& Bock/Boosey \& Hawkes (Berlin).

5. Mentionné par Ulrich Dibelius : Moderne Musik nach 1945, München, Piper³ , 1998, p. 61.

6. L'opus 1 de Blacher est la pièce Vocalises de jazz [Jazz-Koloraturen] pour soprano, saxophone alto et basson en 1929.

7. Cf. Boris Blacher. Dokumente zu Leben und Werk, hg. Marita Gleiss \& Heribert Henrich, Berlin, Stiftung Archiv der Akademie der Künste/Henschel, 1993, p. 31.

8. Pour une vue d'ensemble sur la situation de Blacher, $c f$. Dibelius, op. cit., p. 60 et passim.

9. Il écrit par exemple en 1949, dans une lettre en anglais à William Glock: " The musical life is now quite normal with Furtwängler playing Brahms + Bruckner in the same way as nothing happened in the past. All the hopes that something has changed since 45 are gone. ", in : Boris Blacher. Dokumente zu Leben und Werk, op. cit., p. 89.

10. Cette œuvre née de l'initiative de compositeurs de l'Est et de l'Ouest est une cantate sur un texte de Jens Gerlach, auteur de la République Fédérale d'Allemagne installé en République Démocratique Allemande en 1953. En choisissant pour sujet la révolte dans le ghetto de Varsovie, les cinq compositeurs Blacher, Dessau, Henze, Karl-Amadeus Hartmann et Wagner-Régeny entendaient élever un cri d'alarme contre la recrudescence de l'antisémitisme en Allemagne. La création, prévue à l'automne 1961, fut repoussée à cause de la construction du mur de Berlin. Elle n'eut lieu qu'en 1966. Cf. Thomas Eickhoff und Werner Grünzweig: «Gerty Herzog-Blacher im Gespräch », in : Heribert Henrich, Thomas Eickhoff : Boris Blacher, Hofheim, Wolke, 2003, p. 34 ; Ulrich Dibelius, Frank Schneider, Neue Musik im geteilten Deutschland. Dokumente aus den fünfziger Jahren, Berlin, Henschel, 1993, p. 323-324.

11. Cf. sur ce point Boris Blacher. Dokumente zu Leben und Werk, op. cit., p. 27. Haggars est le nom de la ville natale de Haller en Estonie ; le titre de l'opéra signifie «barbier » en estonien et est une allusion à Pierre le Grand, premier tsar sans barbe.

12. Cf. aussi les Zeitungsausschnitte de Hanns Eisler (1926).

13. Cf. le scandale provoqué par Neues vom Tage de Hindemith en 1929.

14. C'est sans doute l'une des raisons pour lesquelles cet opéra est l'un des rares qui ait été régulièrement repris en Allemagne, notamment dans les années 1960 puis en 1987, 1988 et 1992. Cependant, dans un compte rendu paru dans la presse, le compositeur Bernd Alois Zimmermann juge l'œuvre maladroite et attribue sa faiblesse à son sujet qui semble héritier du vérisme. Peutêtre Zimmermann songe-t-il à la noirceur de l'intrigue du Tabarro de Puccini ; le réalisme des 
situations lui semble contradictoire avec le dépouillement dont témoigne l'œuvre par ailleurs. Cf. Bernd Alois Zimmermann, in : Stimmen. Monatsblätter für Musik 13/14 (1949), p. 390 (archives Bote \& Bock, dossier de presse).

15. $C f$. la discussion entre Brecht et Blacher, rapportée par Brecht dans son Journal à la date du 29 avril 1950, où les deux artistes s'interrogent sur les raisons pour lesquelles le $\mathrm{xx}^{\mathrm{e}}$ siècle n'a pas produit d'opéra ayant les faveurs du public.

16. Étant donné la position d'Egk sous le régime national-socialiste (Egk avait dirigé la Fachschaft Komponisten de la Reichsmusikkammer), la relation entre les deux hommes était sans doute complexe. Cf. Thomas Eickhoff und Werner Grünzweig, op. cit., p. 33-34, et Mösch, op. cit., p. 165-166.

17. Cf. par exemple Wilhelm Herrmann: "Wer ist nun durchgefallen? Mannheimer Theaterskandal um Abstrakte Oper Nr. $1 »$. Westdeutsche Neue Presse, 19 octobre 1953 (archives Bote \& Bock). Deux extraits de la version concertante donnée le 28 juin 1953 sous la direction de Werner Egk sont disponibles en enregistrement (RCA/BMG 74321 73504). On trouve aux archives Bote \& Bock un enregistrement intégral par l'orchestre symphonique de Séoul sous la direction de Hans Jürgen Nagel (sans date).

18. Alors que dans le ballet (par exemple le Ballet triadique de Schlemmer), l'expérimentation est tentée.

19. Cf.Darmstädter Gespräch. Das Menschenbild in unserer Zeit, hg. Hans Gerhard Evers, Darmstadt, Neue Darmstädter Verlagsanstalt 1950, p. 193 et suiv. Adorno emploie métaphoriquement le mot Unversetztheit (utilisé par exemple pour désigner la pureté du vin dans la messe catholique) pour expliciter la rupture d'un lien existant entre la forme et le contenu, entre l'art et le monde à représenter. Voir aussi Hans Heinz Stuckenschmidt: Boris Blacher, Berlin-Wiesbaden, Bote \& Bock, 1981, p. 36 et suivantes.

20. Partition, p. 2.

21. Werner Egk : Die Zeit wartet nicht, München, Goldmann, 1981, p. 450.

22. Cf. l'exemplaire du "livret» dactylographié, qui n'a bien sûr pas été édité séparément (Akademie der Künste, fonds Blacher 1.75.53).

23. Ou des septièmes majeures : do dièse-ré.

24. Et plus généralement, à l'origine, le tétracorde descendant. Cf. Ellen Rosand: "The Descending Tetrachord. An Emblem of Lament ", in : The Musical Quarterly LXV 3 (1979), p. 358.

25. La scène s'achève dans la partition par un la aigu tenu par le ténor seul, mais dans l'enregistrement dirigé par Hans Jürgen Nagel, cette note est chantée aussi par la soprano, ce qui renforce l'association avec un duo d'amour de l'opéra italien.

26. Cf. sur ce point Hunkemöller : Boris Blacher, der Jazz-Komponist, Frankfurt/Main, Peter Lang 1998, p. 66-67. La scène 6, Amour II, utilise le même principe.

27. Un représentant du même phénomène dans la littérature romanesque: le personnage d'Odysseus Cotton dans Pigeons dans l'herbe de Wolfgang Koeppen, qui découvre Munich la radio sur l'épaule, accompagné d'une voix chantant du blues (Tauben im Gras, Frankfurt/Main, Suhrkamp, st 2316, p. 27 et passim).

28. On peut penser que les fautes d'orthographe en anglais sont voulues : il s'agit d'évoquer des mots mais de souligner aussi le peu d'importance du sens. L'hésitation entre la conjonction de subordination "wether » et le substantif « weather » indique également que le dialogue repose sur des fausses phrases, à la grammaire incohérente.

29. Ou bien : " eh, toi, réponds !"

30. La référence dut paraître encore plus précise, sinon trop précise, lors de la création radiophonique à Francfort le 28 juin 1953, donc quelques jours après la répression des manifestations de Berlin-Est par les chars soviétiques le 17 juin.

31. Le lien entre ces syllabes et un signifiant existant est souligné par l'orthographe : le $y$ dans adynazit, qui aurait pu être un ü en allemand, renvoie évidemment au mot « dynamite». 
32. Un rapprochement plus précis serait possible avec ce que Paul Dessau devait mettre en musique vingt ans plus tard dans son opéra Einstein, auquel il commença à travailler avec Brecht dès 1955, immédiatement après la mort du physicien.

33. Le principe de composition avec des mètres variables est l'une des caractéristiques principales de Blacher; cf. Christopher Grafschmidt: Boris Blachers variable Metrik und ihre Ableitungen : Voraussetzungen - Ausprägungen - Folgen, Bern, Peter Lang, 1996.

34. Bertolt Brecht, Werke, Bd. 25, Frankfurt/Main, Surhkamp - Berlin, Aufbau, 1994, p. 79.

35. Kurt Schwitters : Sprache, in : Kurt Schwitters : Eile ist des Witzes Weile, hg. Christina Weiss und Karl Riha, Stuttgart, Reclam 1987, p. 135-137.

36. Cf. le commentaire de Wulf Konold, selon lequel l'œuvre entretient un rapport ironique avec le genre de l'opéra qui, au fur et à mesure de son histoire et de l'utilisation d'un orchestre de plus en plus complexe, rend le texte inintelligible, in : Enzyklopädie des Musiktheaters, Bd. 1, München, Piper, 1986, p. 364.

37. Rien ne permet de savoir si le metteur en scène, Hans Schöne, songeait à la Pièce didactique de Baden-Baden de Brecht/Hindemith où deux clowns interviennent dans la troisième enquête. Toujours est-il que les contrastes entre le sérieux et le comique, entre le naturel et le mécanique, sont parmi les moyens les plus perceptibles de la distanciation.

38. Wulf Konold (op. cit., p. 364) écrit qu'on ne peut savoir si Egk fait allusion à la scène 2 (Amour I) ou à la scène 6 (Amour II). Mais Egk pense implicitement à Amour I, puisque la scène décrite est pour ténor et soprano; dans Amour II, le ténor est seul avec les chœurs.

39. Werner Egk : Die Zeit wartet nicht, op. cit., p. 448-449.

40. Voir notamment la thèse non publiée de Martin Willenbrink, Der Zeitopernkomponist Boris Blacher, Berlin, Technische Universität, 1994.

41. Paris, Denoël, 1939 ; Minuit, 1957 [références données ici d'après cette édition], p. 39.

42. Ingeborg Bachmann : Alle Tage, in : Die gestundete Zeit.Werke 1, hg. Christine Köschel, Inge von Weidenbaum, Clemens Münster, München, Piper, 1978, p. 46.

\section{RÉSUMÉS}

L'article étudie un livret d'un genre bien particulier, celui d' Abstrakte Oper Nr.1 de Boris Blacher (1953). Après un rappel sur ce compositeur injustement négligé par la critique et le concert et sa production lyrique avant Abstrakte Oper Nr.1, il s'agira tout d'abord de replacer cette entreprise dans le cadre des années 1950, assez peu étudiées, ensuite d'analyser les rapports entre texte, musique et scène dans un ouvrage qui tente de transposer dans le domaine du théâtre musical des principes de la peinture abstraite. Le rôle du livret (traditionnellement fait de mot et porteur de l'«intrigue ») est ici paradoxal, car s'il conserve, malgré sa tendance « abstraite ", quelques éléments de langage dramatique, il se dérobe également à la hiérarchie traditionnelle à l'opéra qui fait de ce substrat de la musique un élément second ou secondaire dans la représentation d'opéra. Cependant, cette abstraction même n'exclut pas les rapports à l'histoire et l'œuvre n'est pas sans lien avec les Zeitopern (« opéras d'actualité ») des années 1920.

Der Aufsatz untersucht das höchst eigenartige Libretto von Boris Blachers Abstrakte Oper Nr.1. Zuerst wird Blachers Laufbahn und Opernproduktion vor Abstrakte Oper Nr. 1 im Kontext der 1950er Jahre beschrieben. Dann werden die Beziehungen zwischen Text, Musik und Bühne untersucht: Diese Oper ist ein Versuch, Prinzipien der abstrakten Malerei auf das Musiktheater 
zu übertragen; dabei nimmt das Libretto als eine Art Text, der üblicherweise aus Wörtern besteht und Handlungsträger ist, eine paradoxe Stellung ein, denn es behält trotzdem Spuren des Dramatischen und entzieht sich gleichzeitig der üblichen Hierarchie zwischen den Bestandteilen einer Oper. Schließlich erweist sich diese Abstraktheit auch als eine Möglichkeit, Geschichtliches einzubeziehen. Insofern ist dieses Werk mit den Zeitopern der 1920er Jahre verwandt.

INDEX

Mots-clés : Zeitoper, théâtre musical, peinture oeuvrecitee Abstrakte Oper Nr.1

\section{AUTEURS}

BERNARD BANOUN

Université François-Rabelais, Tours 